\title{
EFEITOS DO THIDIAZURON E DO ÁCIDO GIBERÉLICO NAS CARACTERÍSTICAS DOS CACHOS E BAGAS DE UVAS 'NIAGARA ROSADA' NA REGIÃO DE JUNDIAÍ-SP'
}

\author{
RENATO VASCONCELOS BOTELHO², ERASMO JOSÉ PAIOLI PIRES ${ }^{3}$, MAURILO MONTEIRO TERRA³, CÁSSIA \\ REGINA LIMONTA CARVALHO ${ }^{4}$
}

\begin{abstract}
RESUMO - Dois experimentos foram conduzidos em vinhedo localizado na região de Jundiaí-SP, com o objetivo de estudar-se os efeitos de aplicações de fitorreguladores em uvas 'Niagara Rosada'. No primeiro ensaio, utilizou-se de thidiazuron na concentração de 5 ou $10 \mathrm{mg}$. $\mathrm{L}^{-1}$, e/ou ácido giberélico na concentração de $100 \mathrm{mg} \cdot \mathrm{L}^{-1}$. No segundo experimento, as doses de thidiazuron foram: $0 ; 2,5 ; 5,0 ; 7,5 ; 10,0 ; 12,5$ e $15,0 \mathrm{mg} . \mathrm{L}^{-1}$. Os fitorreguladores foram aplicados mediante única imersão dos cachos em solução, 14 dias após a plena floração. Foram avaliados massa, largura e comprimento dos cachos e bagas; massa dos engaços; número de bagas; número de sementes; teor de sólidos solúveis totais e acidez total titulável do mosto. No primeiro ensaio, os maiores valores de massa, comprimento e largura das bagas foram verificados para os tratamentos com thidiazuron associado a ácido giberélico. O tratamento isolado de ácido giberélico não foi efetivo para aumentar o tamanho das bagas e reduziu o número de bagas por cacho. No segundo experimento, aplicações de thidiazuron aumentaram linearmente a massa de cachos e engaços, e a massa, largura e comprimento das bagas. As variáveis teor de sólidos solúveis totais, acidez total titulável, número de sementes e largura de cachos não foram influenciadas pelo TDZ e pelo $\mathrm{AG}_{3}$.
\end{abstract}

Termos para Indexação: Vitis labrusca, fitorregulador, frutos, giberelina, citocinina.

\section{EFFECTS OF THIDIAZURON AND GIBBERELLIC ACID ON CLUSTER AND BERRIES CHARACTERISTICS OF 'NIAGARA ROSADA' GRAPES IN THE REGION OF JUNDIAÍ-SP}

\begin{abstract}
Two trials were carried out in vineyards located in Jundiaí-SP, with the objective of studying the effects of growth regulators applications on 'Niagara Rosada' grapes. In the first trial, it was used thidiazuron (TDZ) at 5 or 10mg.L $\mathrm{L}^{-1}$, and/or gibbellic acid at 100mg.L $\mathrm{L}^{-1}$. In the second experiment, the following doses of thidiazuron were used: $0 ; 2.5,5.0,7.5,10.0,12.5$ and 15.0mg.L $\mathrm{L}^{-1}$. The growth regulators were applied by dipping the cluster 14 days after full blooming. It was evaluated weight, length and width of clusters and berries; rachis weight; number of berries; number of seeds; total soluble solutes content and total titratable acidity of juice. In the first experiment, the highest weight, length and width of berries were verified to the treatments with thidiazuron associated to gibberellic acid. Treatments with only gibberellic acid was not effective in increasing berry size and reduced number of berries per cluster. In the second trial, thidiazuron applications increased linearly weight of rachis and cluster; and weight, length and width of berries. There weren't significant differences for total soluble solutes content, total titratable acidity, number of seeds and cluster width.
\end{abstract}

Index terms: Vitis labrusca, growth regulator, fruits, gibberellin, cytokinin

\section{INTRODUÇÃO}

A viticultura paulista aparece em posição de destaque no cenário nacional. Com produção anual de 201.630 toneladas, em 11.553 hectares, representa $21 \%$ da produção brasileira, sendo a maior parte do cultivo destinado à produção de uvas de mesa (AGRIANUAL, 2001). Segundo Sousa (1996), entre as variedades cultivadas no Estado de São Paulo, destacam-se as Niagaras Rosada e Branca, com 77,0\% da produção total.

$\mathrm{O}$ ácido giberélico $\left(\mathrm{AG}_{3}\right)$ é o fitorregulador mais utilizado em viticultura, visando principalmente ao aumento do tamanho e fixação das bagas, à descompactação dos cachos e à eliminação de sementes. Com o objetivo de aumentar o tamanho das bagas, as respostas às aplicações ácido giberélico variam em função da cultivar e das condições de cultivo (Pires et al., 1998). No caso das variedades 'Niagara Rosada' e 'Niagara Branca', a maioria dos estudos já realizados não apresentaram efeitos significativos de aplicações de ácido giberélico nas dimensões dos frutos (Pereira et al.,1979; Maraschin et al.,1986).

O thidiazuron (N-fenil-N-1,2,3-tidiazol-5-tiuréia) (TDZ) é um fitorregulador que, no Brasil, é largamente utilizado na cultura do algodoeiro para provocar desfolhamento. Esse produto também vem sendo usado na cultura de tecidos para induzir brotação in vitro, visto que demonstra ter ação semelhante à das citocininas (Petri et al., 1992).

Em fruticultura, há trabalhos que comprovaram a eficiência do thidiazuron na quebra de dormência de gemas de macieira e pereira (Araújo et al., 1991; Francisconi et al., 1992) e no aumento do tamanho e pegamento dos frutos de maçãs, kiwis e caquis (Petri et al., 1992; Schuck
\& Petri, 1992; Itai et al., 1995)

Nas variedades de videiras sem sementes Sovereign Coronation, Simone, Selection 495 e Selection 535, Reynolds et al. (1992) estudaram os efeitos de aplicações de thidiazuron nas doses de 0; 4 e 8 mg.L-1 , quando as bagas atingiram $5 \mathrm{~mm}$ de diâmetro. Pelos resultados obtidos, verificaram que o thidiazuron aumentou linearmente a massa dos cachos e bagas e reduziu o teor de sólidos solúveis totais e o $\mathrm{pH}$ do mosto.

Por outro lado, Byun \& Kim (1995) trataram cachos de videiras da cultivar Kyoho, com $\mathrm{AG}_{3}$ a $20 \mathrm{mg} . \mathrm{L}^{-1}$ e thidiazuron a 5 ou $10 \mathrm{mg} . \mathrm{L}^{-1}, 5$ dias após o pleno florescimento, e verificaram que $\mathrm{AG}_{3}$ aumentou o tamanho das bagas, enquanto o thidiazuron aumentou o número de bagas. Tratamentos combinados de thidiazuron e $\mathrm{AG}_{3}$ aumentaram $\mathrm{o}$ tamanho e o número de bagas. Entretanto, thidiazuron reduziu a coloração das bagas e o teor de sólidos solúveis totais. Resultados semelhantes foram verificados por Schuck (1994), em uvas 'Vênus'.

Tendo em vista as possibilidades de utilização de fitorreguladores para a melhoria da qualidade de uvas, este trabalho teve como objetivo estudar os efeitos de aplicações de thidiazuron e ácido giberélico nas características dos cachos e bagas de uvas da cultivar Niagara Rosada na região de Jundiaí-SP.

\section{MATERIAL E MÉTODOS}

Os experimentos foram conduzidos em vinhedo da cultivar Niagara Rosada, localizado no município de Jundiaí-SP, com coordenadas geográficas de $23^{\circ} 06^{\prime} \mathrm{S}$ e $46^{\circ} 55^{\prime} \mathrm{W}$, e $715 \mathrm{~m}$ de altitude. As videiras em

\footnotetext{
(Trabalho 051/2002). Recebido: 15/03/2002; Aceito para publicação: 26/03/2003. Parte da Tese de Doutorado do primeiro autor apresentada ao curso de pósgraduação em Agronomia da FCA-UNESP, campus de Botucatu-SP.

${ }^{2}$ Eng. Agr. Dr. Centro de Fruticultura, Instituto Agronômico de Campinas, Caixa Postal 28, CEP 13001-970 Campinas-SP. Tel. (19) 3241-9910. Email:rebotelho@uol.com.br Bolsista da FAPESP.

${ }^{3}$ Eng. Agr. Dr., Pesquisador Científico. Instituto Agronômico de Campinas. Bolsistas CNPq

${ }^{4}$ Química, M. Sc. Pesquisadora Científica. Centro de Genética, Biologia Molecular e Fitoquímica. Instituto Agronômico de Campinas.
} 
$18^{\circ}$ ano de produção estavam enxertadas sobre porta-enxerto IAC-766 Campinas, espaçadas em 2,20x1,00 m, e conduzidas no sistema de espaldeira.

No primeiro experimento, os tratamentos foram os seguintes: T1 - Testemunha;

T2- TDZ a $5 \mathrm{mg} . \mathrm{L}^{-1}$;

T3 - TDZ a 10mg.L-1

T4 - TDZ a $5 \mathrm{mg} . \mathrm{L}^{-1}+\mathrm{AG}_{3}$ a $100 \mathrm{mg} . \mathrm{L}^{-1}$;

T5-TDZ a $10 \mathrm{mg} . \mathrm{L}^{-1}+\mathrm{AG}_{3}$ a $100 \mathrm{mg} . \mathrm{L}^{-1}$;

$\mathrm{T} 6-\mathrm{AG}_{3}$ a $100 \mathrm{mg} \cdot \mathrm{L}^{-1}$.

No segundo experimento, os tratamentos consistiram das seguintes doses de thidiazuron: $0 ; 2,5 ; 5,0 ; 7,5 ; 10,0 ; 12,5$ e $15,0 \mathrm{mg} . \mathrm{L}^{-1}$.

Todas as práticas culturais no vinhedo, exceto a utilização de ácido giberélico, foram idênticas ao sistema convencional da propriedade para toda a área experimental.

Os tratamentos com fitorreguladores foram efetuados aos 14 dias após o pleno florescimento, através de única imersão dos cachos na solução contida em recipiente plástico, adicionada de espalhante adesivo Iharaguen-S ${ }^{\circledR}$ a $1 \%$, formulação comercial com $20 \%$ de polioxietileno alquilfenol éter. Para o preparo das soluções com reguladores de crescimento, utilizaram-se os produtos comerciais Dropp ${ }^{\circledR}$, com $50 \%$ de thidiazuron e Pro-Gibb ${ }^{\circledR}$, com $10 \%$ de ácido giberélico.

Em todos os experimentos, o delineamento experimental foi em blocos casualizados, com 6 repetições e 2 cachos por parcela. Videiras semelhantes em vigor foram selecionadas para cada bloco, na mesma linha de plantio, e os cachos foram sorteados dentro de cada um dos 6 blocos, para a realização dos tratamentos.

A coleta dos cachos foi realizada no dia 22 de dezembro de 1999, quando o tratamento-testemunha atingiu o ponto de colheita comercial, ou seja, com teor de sólidos solúveis totais mínimo de $14,0^{\circ} \mathrm{Brix}$ (CEAGESP, 2000). Posteriormente, os cachos foram acondicionados em sacos de polietileno, devidamente identificados, transportados em caixas plásticas para o Instituto Agronômico de Campinas, onde foram mantidos em câmara frigorífica, à temperatura de $3^{\circ} \mathrm{C}$, durante o período de avaliação.

Foram determinadas as seguintes variáveis:

1. Massa dos cachos, bagas e engaços, em balança de precisão. Após a medição da massa dos cachos, as bagas foram separadas dos pedicelos, cortando-os com tesoura, para posterior pesagem das bagas e dos engaços, separadamente.

2. Comprimento e largura dos cachos e bagas, com paquímetro digital. Para as medições das dimensões das bagas, utilizou-se de uma amostra de 20 bagas por cacho, calculando-se, posteriormente, a média de cada amostra.

3. Número de bagas por cacho.

4. Número de sementes por baga (subamostra de 20 bagas).

Para cada parcela, foram realizadas as seguintes análises do mosto de 100 bagas:

1.Teor de sólidos solúveis totais: com auxílio de refratômetro de mesa com autocompensação de temperatura (Carvalho et al., 1990);

2. Acidez total titulável: por titulação em uma alíquota de $5 \mathrm{ml}$ do mosto com $\mathrm{NaOH} 0,1 \mathrm{~N}$, e expressa em g de ácido tartárico por $100 \mathrm{ml}$ de mosto (Carvalho et al., 1990).

No experimento 1, em que se estudaram os efeitos de aplicações de thidiazuron e ácido giberélico, os resultados foram submetidos à análise de variância e comparação de médias pelo teste de Duncan, ao nível de $5 \%$ de probabilidade. Para os dados do experimento 2 , em que foram estudados os efeitos de doses crescentes de thidiazuron, efetuou-se a análise de variância, estudando-se a regressão através dos polinômios ortogonais.

\section{RESULTADOS E DISCUSSÃO}

\section{Experimento 1}

Todos os tratamentos em que se aplicou thidiazuron, combinado ou não com ácido giberélico, aumentaram a massa dos cachos e os engaços (Tabela 1), estando de acordo com outros resultados de pesquisa ( Reynolds et al., 1992; Byun \& Kim, 1995 e Schuck, 1994).
Tendências semelhantes foram verificados para os dados de comprimento e largura dos cachos, embora não tenha havido aumentos significativos para a maioria dos tratamentos com fitorreguladores (Tabela 1).

TABELA 1-Massa, comprimento e largura dos cachos, e massa dos engaços de uvas da cultivar Niagara Rosada tratadas com thidiazuron e ácido giberélico (Jundiaí-SP, 1999).

\begin{tabular}{|c|c|c|c|c|}
\hline Tratamentos & $\begin{array}{c}\text { Massa } \\
\text { Cachos (g) }\end{array}$ & $\begin{array}{c}\text { Comprimento } \\
\text { cachos }(\mathrm{cm})\end{array}$ & $\begin{array}{l}\text { Largura } \\
\text { cachos }(\mathrm{cm})\end{array}$ & $\begin{array}{c}\text { Massa } \\
\text { Engaços(g) }\end{array}$ \\
\hline Testemunha & $209,17 c^{1}$ & 11,69 & $7,18 \mathrm{~b}$ & 1,87 \\
\hline TDZ 5 mg.L L $^{-1}$ & $284,00 \mathrm{a}$ & 12,83 & $9,37 \mathrm{a}$ & $2,85 \mathrm{a}$ \\
\hline TDZ $10 \mathrm{mg} \cdot \mathrm{L}^{-1}$ & $259,00 \mathrm{~b}$ & 13,08 & $7,80 \mathrm{~b}$ & $2,83 \mathrm{a}$ \\
\hline TDZ 5mg.L ${ }^{-1}+\mathrm{AG}_{3}$ & $241,33 \mathrm{~b}$ & 12,98 & $7,82 \mathrm{~b}$ & $2,48 \mathrm{ab}$ \\
\hline TDZ 10 mg. $\mathrm{L}^{-1}+\mathrm{AG}_{3}$ & $255,33 \mathrm{~b}$ & 11,85 & $8,12 \mathrm{ab}$ & $2,31 \mathrm{bc}$ \\
\hline $\mathrm{AG}_{3}$ & $202,83 \mathrm{c}$ & 11,73 & $7,22 \mathrm{~b}$ & $1,93 \mathrm{c}$ \\
\hline CV (\%) & 7,93 & 9,62 & 14,74 & 16,20 \\
\hline
\end{tabular}

${ }^{1}$ Médias seguidas pela mesma letra, na mesma coluna, não diferem entre si, pelo teste de Duncan, ao nível de $5 \%$ de probabilidade.

A massa, comprimento e largura das bagas foram igualmente influenciados pelas aplicações de fitorreguladores, sendo que os maiores aumentos para estas variaveis foram verificados quando se associaram thidiazuron e ácido giberélico (Tabela 2). A maior massa das bagas foi observada para o tratamento com thidiazuron a $10 \mathrm{mg} . \mathrm{L}^{-1}$, associado a ácido giberélico a $100 \mathrm{mg} . \mathrm{L}^{-1}$, conferindo um aumento de 37,6\% em relação à testemunha. Aplicações isoladas de ácido giberélico foram pouco eficientes para promover o aumento do tamanho das bagas, estando de acordo com os resultados de Pereira et al. (1979) e de Maraschin et al. (1986).

O maior desenvolvimento das bagas e engaços promovido por aplicações de thidiazuron pode ser explicado pela sua ação de citocinina em tecidos vegetais, induzindo a divisão celular, em geral, por uma interação com auxinas (Mcgraw, 1988). Além disso, segundo Davies (1988), citocininas podem estimular o crescimento celular e retardar a senescência de órgãos vegetais.

Aparentemente, aplicações de thidiazuron aumentaram o número de bagas, enquanto o ácido giberélico apresentou efeito inverso, reduzindo o número de bagas por cacho. Os cachos tratados com thidiazuron a 5 e $10 \mathrm{mg} . \mathrm{L}^{-1}$ apresentaram, respectivamente, 32,7 e $23,8 \%$ mais bagas do que aqueles tratados com ácido giberélico (Tabela 2). Para o tratamento-testemunha e aqueles em que foram associados os dois fitorreguladores, os valores para esta variável foram intermediários.

TABELA 2-Massa, comprimento, largura e número de bagos de uvas da cultivar Niagara Rosada tratadas com thidiazuron e ácido giberélico (Jundiaí-SP, 1999).

\begin{tabular}{|c|c|c|c|c|}
\hline Tratamentos & $\begin{array}{c}\text { Massa } \\
\text { bagos (g) }\end{array}$ & $\begin{array}{c}\text { Comprimento } \\
\text { bagos (mm) }\end{array}$ & $\begin{array}{c}\text { Largura } \\
\text { bagos }(\mathrm{mm})\end{array}$ & $\begin{array}{c}\text { Número } \\
\text { Bagos/cacho }\end{array}$ \\
\hline Testemunha & $4,07 \mathrm{c}^{1}$ & $20,22 \quad c$ & $17,95 \mathrm{c}$ & $51,2 \mathrm{abc}$ \\
\hline TDZ $5 \mathrm{mg} . \mathrm{L}^{-1}$ & $5,18 \mathrm{ab}$ & $21,16 a b$ & $19,03 \mathrm{~b}$ & $57,5 \mathrm{a}$ \\
\hline TDZ $10 \mathrm{mg} \cdot \mathrm{L}^{-1}$ & $4,77 \mathrm{~b}$ & $21,44 a b$ & $19,16^{\mathrm{a} b}$ & $53,6 \mathrm{ab}$ \\
\hline TDZ $5 \mathrm{mg} \cdot \mathrm{L}^{-1}+\mathrm{AG}_{3}$ & $5,06 \mathrm{ab}$ & $21,88 \mathrm{a}$ & $19,83^{\mathrm{a}}$ & $47,3 \mathrm{bc}$ \\
\hline TDZ 10 mg. $\mathrm{L}^{-1}+\mathrm{AG}_{3}$ & $5,60 \mathrm{a}$ & $21,82 \mathrm{a}$ & $19,72^{\mathrm{a}}$ & $45,0 \mathrm{c}$ \\
\hline $\mathbf{A G}_{3}$ & $4,66 \mathrm{bc}$ & $20,81 \mathrm{bc}$ & $18,70 \mathrm{~b}$ & $43,3 \mathrm{c}$ \\
\hline CV $(\%)$ & 10,83 & 10,89 & 2,84 & 12,55 \\
\hline
\end{tabular}

${ }^{1}$ Médias seguidas pela mesma letra, na mesma coluna, não diferem entre si, pelo teste de Duncan, ao nível de 5\% de probabilidade.

Aumento do número de bagas causado por aplicações de thidiazuron também foi relatado por Byun \& Kim (1995) em uvas da cultivar Kyoho. Segundo Hayata et al. (2000), substâncias com ação de citocinina podem promover o pegamento de frutos devido ao seu efeito em atrair nutrientes para os órgãos tratados, aumentando a habilidade dos frutos jovens em competir por assimilados com o resto da planta.

Por outro lado, aplicações de ácido giberélico poderiam ter le- 
vado à degrana dos cachos. Pérez \& Morales (1999) constataram que a atividade da peroxidase solúvel dos pedicelos de uvas 'Sultana' aumentou significativamente com o incremento das doses de ácido giberélico aplicado em pós-florescimento nas videiras, sugerindo o possível envolvimento desta enzima na lignificação de pedicelos e ráquis causados pela aplicação de $\mathrm{AG}_{3}$. Esta lignificação dos pedicelos poderia, portanto, levar à perda de flexibilidade dos pedicelos e, conseqüentemente, provocar a degrana dos cachos.

As aplicações de fitorreguladores não apresentaram efeito significativo para as variáveis teor de sólidos solúveis totais e acidez total titulável do mosto e número de sementes por baga (Tabela 3). Estes resultados não são condizentes com os estudos de Reynolds et al. (1992), Schuck (1994) e Byun \& Kim (1995), que verificaram redução do teor de sólidos solúveis totais e $\mathrm{pH}$, e aumento da acidez total titulável do mosto de uvas tratadas com thidiazuron. Possivelmente, esta diferença de resultados está relacionada a uma característica varietal. De acordo com Pires et al. (1998), as respostas às aplicações de fitorreguladores variam em função da cultivar e das condições de cultivo.

TABELA 3- Teor de sólidos solúveis totais e acidez total titulável do mosto, e número de sementes por bago de uvas da cultivar Niagara Rosada tratadas com thidiazuron e ácido giberélico (Jundiaí-SP, 1999).

\begin{tabular}{lccc}
\hline Tratamentos & $\begin{array}{c}\text { Sólidos solúveis } \\
\text { totais (\%) }\end{array}$ & $\begin{array}{c}\text { Acidez total } \\
\text { titulável }(\mathbf{g} / \mathbf{1 0 0 m l})\end{array}$ & $\begin{array}{c}\text { Número } \\
\text { sementes/ } \\
\text { bago }\end{array}$ \\
\hline Testemunha & 14,31 & 0,852 & 2,88 \\
TDZ 5 mg.L ${ }^{-1}$ & 14,85 & 0,755 & 3,07 \\
TDZ 10 mg.L & 14,36 & 0,792 & 3,12 \\
TDZ 5 mg.L ${ }^{-1}+\mathbf{A G}_{3}$ & 14,53 & 0,810 & 2,82 \\
TDZ 10 mg.L ${ }^{-1}+\mathbf{A G}_{\mathbf{3}}$ & 14,38 & 0,835 & 3,03 \\
$\mathrm{AG}$ & 14,72 & 0,807 & 2,93 \\
CV (\%) & 3,80 & 10,83 & 10,56 \\
\hline
\end{tabular}

\section{Experimento 2}

No segundo experimento, em que se estudou o efeito de doses crescentes de thidiazuron, verificou-se que houve aumento linear para as variáveis massa de cachos e engaços (Figura 1), e massa, comprimento e largura das bagas (Figura 2).

O aumento da massa e dimensões das bagas, e da massa dos engaços, possivelmente, está diretamente relacionado à ação de citocinina do thidiazuron, levando ao aumento da divisão e expansão celular (Mcgraw, 1988; Davies, 19f88), estando de acordo com os resultados de Reynolds et al. (1992), em 4 variedades de uvas sem sementes.

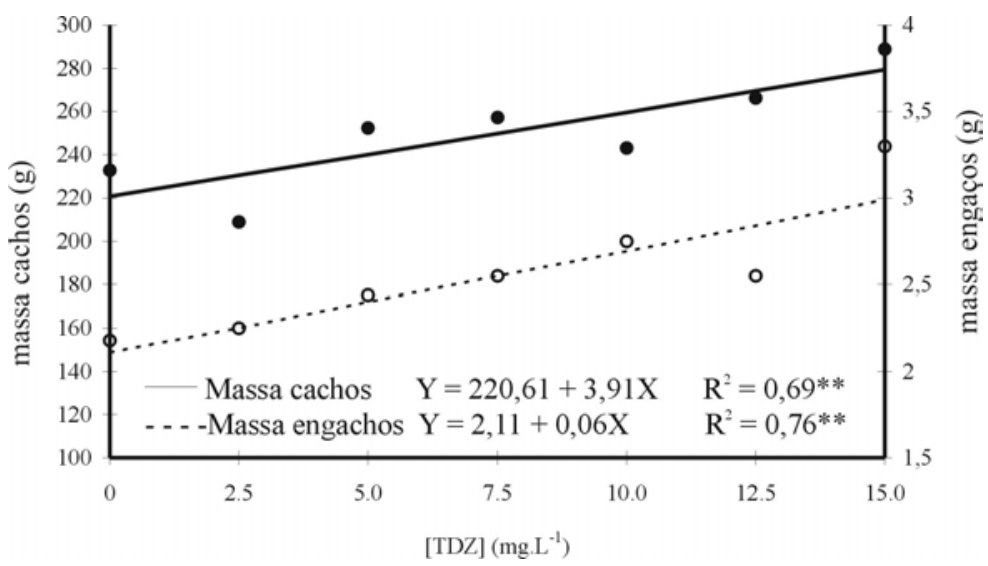

FIGURA 1- Massa dos cachos (g) e engaços (g) de uvas 'Niagara Rosada' tratadas com thidiazuron (Jundiaí-SP, 1999).

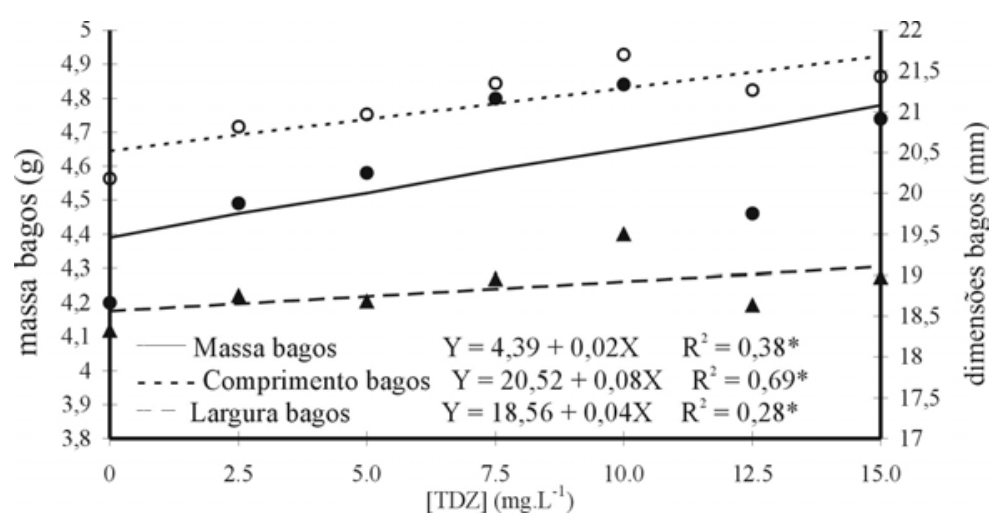

FIGURA 2- Massa (g), comprimento (mm) e largura ( $\mathrm{mm}$ ) dos bagos de uvas 'Niagara Rosada' tratadas com thidiazuron (JundiaíSP, 1999).

No entanto, a ausência de diferenças para as variáveis teor de sólidos solúveis totais e acidez total titulável (Tabela 4), não foi condizente com os relatos de Reynolds et al. (1992) e Byun \& Kim (1995), que observaram atraso na maturação dos frutos tratados com thidiazuron e conseqüente redução do teor de sólidos solúveis totais e aumento da acidez do mosto de uvas. Características climáticas, culturais e varietais podem ser algumas das possíveis causas para as diferenças entre resultados.

O número de sementes por baga e o número de bagas por cacho também não foram influenciados pelas aplicações de thidiazuron. Entretanto, verificou-se ligeira tendência de aumento do comprimento e largura dos cachos tratados com thidiazuron (Tabela 4).

TABELA 4-Comprimento e largura de cachos, número de bagos, número de sementes, teor de sólidos solúveis totais e acidez total titulável do mosto de uvas da cultivar Niagara Rosada tratadas com thidiazuron (Jundiaí-SP, 1999).

\begin{tabular}{|c|c|c|c|c|c|c|}
\hline $\begin{array}{c}\text { Tratamentos } \\
\text { (doses de TDZ) }\end{array}$ & $\begin{array}{c}\text { Comprimento } \\
\text { cachos }(\mathbf{c m})\end{array}$ & $\begin{array}{c}\text { Largura } \\
\text { Cachos }(\mathrm{cm})\end{array}$ & $\begin{array}{l}\text { Número } \\
\text { Bagos }\end{array}$ & Número sementes & SST (\%) & $\begin{array}{c}\text { ATT } \\
(\mathrm{g} / \mathbf{1 0 0 \mathrm { ml } )}\end{array}$ \\
\hline 0mg. $\mathrm{L}^{-1}$ & 12,48 & 7,75 & 55,3 & 2,87 & 14,87 & 0,812 \\
\hline $2,5 \mathrm{mg} \cdot \mathrm{L}^{-1}$ & 11,42 & 7,77 & 46,2 & 3,25 & 15,08 & 0,820 \\
\hline $5,0 \mathrm{mg} \cdot \mathrm{L}^{-1}$ & 12,57 & 8,05 & 55,0 & 2,83 & 13,98 & 0,840 \\
\hline $7,5 \mathrm{mg} . \mathrm{L}^{-1}$ & 12,62 & 7,63 & 56,2 & 2,87 & 14,65 & 0,762 \\
\hline $10,0 \mathrm{mg} . \mathrm{L}^{-1}$ & 12,55 & 8,37 & 49,8 & 2,85 & 14,35 & 0,820 \\
\hline $12,5 \mathrm{mg} \cdot \mathrm{L}^{-1}$ & 13,22 & 8,38 & 58,0 & 2,87 & 15,05 & 0,812 \\
\hline $15,0 \mathrm{mg} . \mathrm{L}^{-1}$ & 12,70 & 8,52 & 60,8 & 2,98 & 14,40 & 0,730 \\
\hline C.V. $(\%)$ & 13,25 & 14,42 & 16,56 & 11,99 & 3,77 & 10,56 \\
\hline
\end{tabular}

Pelos resultados apresentados neste trabalho, ficou evidente a possibilidade de utilização de thidiazuron para o aumento do tamanho das bagas de uvas 'Niagara Rosada'. Para as condições deste experimento, como houve efeito linear para as variáveis estudadas, a dose de $15 \mathrm{mg} . \mathrm{L}^{-1}$ de thidiazuron seria a mais indicada para a obtenção dos maiores aumentos do tamanho de bagas. A associação de ácido giberélico a $100 \mathrm{mg} . \mathrm{L}^{-1}$ aumentou o efeito do thidiazuron no tamanho das bagas; entretanto, ficou demonstrado seu efeito prejudicial na redução do número de bagas por cacho.

O thidiazuron é um fitorregulador que ainda não está registrado 
para a cultura da videira no Brasil, não podendo ser recomendado para o uso de viticultores, porém já está registrado para diversas espécies frutíferas, incluindo a videira, em países como Chile e México. Novas pesquisas, incluindo estudos toxicológicos, deverão ser conduzidas para uma futura aplicação comercial desta substância na fruticultura nacional.

\section{CONCLUSÕES}

1) Aplicações de thidiazuron na concentração de $15 \mathrm{mg} \cdot \mathrm{L}^{-1}, 14$ dias após a floração, aumentam a massa, o comprimento e a largura das bagas de uvas 'Niagara Rosada' na região de Jundiaí (SP).

2) A associação de ácido giberélico a $100 \mathrm{mg} \cdot \mathrm{L}^{-1}$ potencializa o efeito do thidiazuron nas dimensões das bagas de uvas 'Niagara Rosada'.

3) Não há efeito dos fitorreguladores estudados no teor de sólidos solúveis totais e acidez total titulável.

\section{AGRADECIMENTOS}

Agradecemos a colaboração e dedicação do Sra. Maria das Graças dos Santos Lima na condução deste experimento.

\section{REFERÊNCIASBIBLIOGRÁFICAS}

AGRIANUAL 2001. São Paulo: FNP Consultoria \& Comércio, 2001.p.532545.

ARAÚJO, M.M.; FORTES, F.R.L.; SANTOS FILHO, B.G.S. Thidiazuron - uma alternativa para superar a dormência de gemas de macieira (Malus domestica Borkh). Revista Brasileira de Fruticultura, Cruz das almas, v.13, n.3, p.249-253, 1991.

BYUN, J.K.; KIM, J.S. Effects of GA, thidiazuron and ABA on fruit set and quality of 'Kyoho' grapes. Journal of the Korean Society for Horticultural Science, Kyongsan, v.36, n.2, p.231-239, 1995.

CARVALHO, C.R.L.; CARVALHO, P.R.N.; MANTOVANI, D.M.B.; MORAES, R.M. Análise química de alimentos. Campinas: ITAL, 1990.121p.

CEAGESP. Classificação da uva (Vitis vinifera L.). São Paulo: CQH/ CEAESP, 2000. (Programa brasileiro para melhoria dos padrões comerciais e embalagens de hortigranjeiros)

DAVIES, P.J. The plant hormones: their nature, occurrence, and functions. In: _. Plant hormones and their role in plant growth and development. 2.ed. Dordrecht: Kluwer Academic Publishers, 1988. p.1-11.

FRANCISCONI, A.H.D.; BARRADAS, C.I.N.; MARODIN, G.A.B.;
SEIBERT, E. Efeito de óleo mineral, cianamida hidrogenada e thidiazuron na quebra de dormência e produção da pereira (Pyrus communis L.) cv. Packham's Triumph. Revista Brasileira de Fruticultura, Cruz das Almas, v.14, n.1, p.161-166, 1992.

HAYATA, Y.; YOSHIODA, C.; NIIMI, Y.; XINXAN, L. Effects of CPPU on the growth, sugar accumulation and activity of related enzimes in melon fruti. Acta Horticulturae, Leiden, n.514, p.219-225, 2000.

ITAI, A.; TANABE, K.; TAMURA, F.; SUSAKI, S.; YONEMORI, K.; SUGIURA, A. Synthetic cytokinins control persimmon fruit shape, size and quality. Journal of Horticultural Science, Ashford, v.70, n.6, p.867-873, 1995.

MACGRAW, B.A. Cytokinin biosynthesis and metabolism. In: DAVIES, P.J. Plant hormones and their role in plant growth and development. 2.ed. Dordrecht: Kluwer Academic Publishers, 1988. p.76-93.

MARASCHIN, M.; GUERRA, M.P.; SILVA, A.L. Efeitos do ácido giberélico e ethephon sobre as características dos cachos e frutos da cv. Niagara Branca (Vitis labrusca L.) Revista Brasileira de Fruticultura, Cruz das Almas, v.8, n.2, p.51-57, 1986.

PEREIRA, F.M.; SIMÃO, S.; MARTINS, F.P.; IGUE, T. Efeitos da giberelina sobre cachos da cultivar de videira Niagara Rosada. Científica, Jaboticabal, v.7, n.1, p.53-58, 1979.

PÉREZ, F.J.; MORALES, V. A basic preoxidase isoenzyme from the grape pedicel is induced by gibberellic acid. Australian Journal of Plant Physiology, Melbourne, v.26, p.387-390, 1999.

PETRI, J.L.; ARGENTA, L.C.; SUZUKI, A. Efeitos do thidiazuron no tamanho e desenvolvimento dos frutos da macieira. Revista Brasileira de Fruticultura, Cruz das Almas, v.14, n.2, p.127-134, 1992.

PIRES, E.J.P. Emprego de reguladores de crescimento em viticultura tropical. Informe Agropecuário, Belo Horizonte, v.19, n.194, p.40-57, 1998.

REYNOLDS, A.G.; WARDLE, D.A.; ZUROWSKI, C.; LOONEY, N.E. Phenylureas CPPU and thidiazuron affect yield components, fruit composition, and storage potential of four seedless grape selections. Journal of the American Society for Horticultural Science, Alexandria, v.117, p.85-89, 1992.

SCHUCK, E.; PETRI, J.L. Efeitos do thidiazuron no peso médio dos frutos de quivi. Revista Brasileira de Fruticultura, Cruz das Almas, v.14, n.2, p.185-188, 1992.

SCHUCK, E.; Efeitos de reguladores de crescimento sobre o peso dos cachos, bagas e maturação da uva de mesa cv. "Vênus". Revista Brasileira de Fruticultura, Cruz das Almas, v.16, n.1, p.295-306, 1994. SOUSA, J.S.I. Uvas para o Brasil. Piracicaba: FEALQ, 1996. 79 